\title{
Applications of Mathematical Models of Road Cycling
}

\author{
Thorsten Dahmen* Stefan Wolf** Dietmar Saupe* \\ * Department of Computer and Information Science \\ University of Konstanz, 78457 Konstanz, Germany \\ Email: (thorsten.dahmen/dietmar.saupe)@uni-konstanz.de \\ ** inuTech GmbH, 68165 Mannheim, Germany \\ E-mail: stefan.wolf@inutech.de
}

\begin{abstract}
:
This contribution discusses several use cases of mathematical models for road cycling. A mechanical model for the pedaling forces is the basis for an accurate indoor ergometer simulation of road cycling on real-world tracks. Together with a simple physiological model for the exertion of the athlete as a function of his/her accumulated power output, an optimal riding strategy for time trials on mountain ascents is computed. A combination of the two models leads to a mathematical optimization problem that can be solved numerically by discretization. The physical model depends sensitively on an accurate estimation of the road slope on the course. For this purpose, we also present a new method that combines model-based slope estimations with noisy measurements from multiple GPS signals of differing quality. Altogether, we provide a means to simulate road cycling on real-world tracks, to analyze cyclist performance, to identify and quantify potential performance improvement, as well as to instruct the athlete exactly where and how to change his/her pacing strategy to achieve these gains.
\end{abstract}

Keywords: Road Cycling, Cycling Simulators, Optimization of Pacing Strategies, Parameter Estimation, Height Profile Estimation

\section{INTRODUCTION}

Mathematical models are at the core of much research in sport science. They provide the means to simulate individual athletic or team sport performance, to analyze human movement in game sports, to predict and improve performance, to name just a few. In this paper, we provide an introduction and overview of our recent research on applications of mathematical modeling for road cycling.

Our work is oriented towards two primary and one auxiliary goals. The first one is the development of an indoor simulator. The physical setup is based on a programmable ergometer measuring power output of a cyclist. Together with measurements of bike/rider parameters and distances/altitudes along a real-world track we apply a mechanical model for road cycling to control the ergometer which delivers an accurate simulation of a particular athlete's ride on the measured track. Such simulators can be used for realistically training and acquainting athletes with unfamiliar tracks, e.g., for some upcoming racing event at a location too far away for traveling. Moreover, such simulators bring studies in training and sport science, that must be carried out in a lab environment, a step closer to reality. Field studies may not be possible for some experiments because they may require, e.g., regularly taking blood probes to measure lactate concentration.

The second main goal is to optimize rider performance for an uphill time trial, assuming the track has a varying slope profile along the course. For that purpose we propose to additionally apply a simple physiological model of athlete performance that relates the rider's exhaustion to the energy consumed so far. The objective then is to prescribe a strategy to optimally distribute the rider's available energy such that the rider is completely exhausted at the finish line of the time trial and such that the required time is minimal. Our preliminary study indicated that indeed a small performance increase is possible in practice using our simulator for an evaluation.

Our third application of modeling lies in the estimation of parameters of the models. For example, coefficients specifying aerial drag and rolling resistance are required for our applications for simulation and optimization. Typically, such parameters are obtained by linear regression, i.e., in our case by making field measurements of output power while cycling on a known flat track with constant speed and then determining the model parameters such that the predicted power best matches the measured data. However, not all physical parameters can be determined in this way. In our method we propose appropriate compound parameters all of which can be estimated. Moreover, the method allows to make the measurement on tracks that need not be perfectly flat and even significant variations in speed along the measurement rides do not invalidate the experiments.

In case of road cycling in mountainous terrain the dominating factor determining the required power to overcome the forces of gravity and to reach a desired speed is the slope of the track. Thus, together with the mechanical 
cycling model and the rider and bike parameters an accurate road height respectively slope profile is required. We found that current standard GPS measurements of elevation do not provide sufficient accuracy for our application. This can be attributed to the amplification of measurement errors that takes place when numerically differentiating height profiles to compute road gradients. Differential GPS devices are based on correcting ordinary GPS measurements using a reference signal of a fixed base station. With current technology, altitudes measured by differential GPS are exact up to just a few centimeters. However, such devices are prohibitively expensive when compared to other devices required for cycling studies like bicycle computers and even power meters. Moreover, with our differential GPS device, we encountered the disadvantage that common obstacles like trees and houses along the roadside may deteriorate the signal quality to the point that it becomes useless. To fill in such gaps and to generally improve the quality of measured height profiles, we propose to extend the standard procedure for parameter estimation by linear regression of model predictions to measurement data to also approximate entire road slope profiles. Of course, the resulting geometrical road model is incorporated in the computations for the simulation and optimization mentioned previously.

In the following we begin by pointing out the state-ofthe-art regarding parameter estimation, simulation, and optimization of pacing strategies in cycling and related sports. The mechanical bicycling model is at the heart of our applications and explained next in Section 3. Then, we give details for the three main topics of our contribution: simulation, optimization and estimation of road height profile and other parameters (Sections 4 to 6 ). We conclude with an outlook on remaining open problems. For this exposition and review we partially follow our previous publications on the road cycling simulator [Dahmen et al. (2011)], performance optimization [Wolf and Dahmen (2010)], and calibrating model parameters [Dahmen and Saupe (2011)].

\section{PREVIOUS WORK}

Beginning in the 1980s, mathematical models have been developed to describe the relation between pedaling power and speed during road cycling [di Prampero et al. (1979); Olds et al. (1993, 1995)]. These models were derived from the equilibrium of energy demands and supplies. Later Martin et al. (1998) summarized the significant components to form a mathematical model for road cycling power that still serves as the state-of-the-art today and will be outlined in the following section.

Such mathematical models were used to predict time trial performance [Olds et al. (1995)], required power output during cycling [Martin et al. (1998)], and to derive optimal pacing strategies for time trials in variable synthetic terrain and wind conditions [Maronski (1994); Atkinson and Brunskill (2000); Gordon (2005); Atkinson et al. (2007)]. Behncke (1997) reported mathematically advanced related work with emphasis on running which Andreeva and Behncke (1998) extended to hilly tracks.

The models for cycling depend on quite a number of physical parameters. Some of these parameters like the gravity factor of Earth are well known, while others can be measured directly. Existing techniques for the estimation of the remaining model parameters can be divided into three categories: numerical simulations, laboratory experiments, and on-road experiments. For example, simulations by computational fluid dynamics may replace elaborate and expensive wind tunnel tests for determining drag coefficients [Defraeye et al. (2010)]. Also the mathematical models were applied in on-road measurements to identify aerodynamic drag and other physical parameters like rolling friction [e.g., Martin et al. (2006); Lim et al. (2011)]. Not all parameters can be isolated and identified by such on-road measurements. However, compound coefficients that combine these physical parameters, can be determined in this way, leading to an overall improvement of model predictions [Dahmen and Saupe (2011)].

Mathematical models also provide the means upon which ergometers for road biking simulation must be based. In ergometers using an eddy current brake the applied brake force is computed as a function of various mechanical friction parameters, the current speed, and the road slope at the current position in a simulation. Regarding such road cycling simulators there does not seem to exist a detailed account of how to implement the appropriate model equations together with the respective hardware design other than our work in Dahmen et al. (2011). There are commercial ergometers that include a simulation component. However, those are primarily intended for training and entertainment purposes and, thus, not covered in the scientific literature.

\section{MECHANICAL BICYCLING MODEL}

The state-of-the art mathematical model for road cycling power, established by Martin et al. (1998) is a nonlinear differential equation based on an equilibrium of resistance power and pedaling power $P_{\text {ped }}$ provided by a cyclist to propel his bicycle. The resistance power is composed of power due to gain in potential energy $P_{\text {pot }}$, aerodynamic $\operatorname{drag} P_{\text {air }}$, frictional losses in wheel bearings $P_{\text {bear }}$, rolling friction $P_{\text {roll }}$, and gain in kinetic energy $P_{\text {kin }}$ :

$$
P_{\text {pot }}+P_{\text {air }}+P_{\text {bear }}+P_{\text {roll }}+P_{\text {kin }}=\eta P_{\text {ped }} .
$$

The efficiency factor $\eta$ accounts for frictional loss in the drive chain.

Dividing this equation by the angular speed of the wheels yields the corresponding equilibrium of torques where one has to consider the lever principle using the transmission ratio $\gamma=\frac{n_{\text {front }}}{n_{\text {rear }}}$, i.e., the ratio of the number of teeth on the front sprocket to the number of teeth on the rear sprocket:

$$
T_{\text {pot }}+T_{\text {air }}+T_{\text {bear }}+T_{\text {roll }}+T_{\text {kin }}=\frac{\eta}{\gamma} T_{\text {ped }} .
$$

The pedal torque is equal to the product of the pedal force and the length of the crank: $T_{\text {ped }}=F_{\text {ped }} l_{c}$. Moreover, Equation (1) is divided by the radius of the wheels, $r_{w}$, in order to obtain the equilibrium of pedaling and resistance force $F_{\text {resist }}$ acting at the contact area between the rear wheel and the road:

$$
F_{\text {resist }}=F_{\text {pot }}+F_{\text {air }}+F_{\text {bear }}+F_{\text {roll }}+F_{\text {kin }}=\frac{\eta}{\gamma} \frac{l_{c}}{r_{w}} F_{\text {ped }} \text {. }
$$

Eventually, the specific mechanical models can be substituted into each component: 


$$
\begin{gathered}
F_{\text {resist }}=\underbrace{m g \frac{\mathrm{d} h}{\mathrm{~d} x}}_{F_{\text {pot }}}+\underbrace{\frac{1}{2} c_{d} \rho A \dot{x}^{2}}_{F_{\text {air }}}+\underbrace{\left(\beta_{0}+\beta_{1} \dot{x}\right)}_{F_{\text {bear }}}+\underbrace{\mu m g}_{F_{\text {roll }}} \\
+\underbrace{\left(m+\frac{I_{w}}{r_{w}^{2}}\right) \ddot{x}}_{F_{\text {kin }}}=\frac{\eta}{\gamma} \frac{l_{c}}{r_{w}} F_{\text {ped }} .
\end{gathered}
$$

Here, $x=x(t)$ is the distance traveled as a function of time $t, \dot{x}=v(t)$ is the speed, and $\ddot{x}=\dot{v}(t)$ is the acceleration. The other physical parameters are listed in the table below. Ambient wind and aerodynamic drag by rotation of spokes is neglected. For numerical values and details see Dahmen et al. (2011).

\begin{tabular}{llll}
\hline \multicolumn{1}{c}{ Cyclist and bicycle } & & \multicolumn{2}{l}{ Course and environment } \\
\hline total mass (cyclist, bike) & $m$ & friction factor & $\mu$ \\
wheel circumference & $c_{w}$ & gravity factor & $g$ \\
wheel radius & $r_{w}$ & drag coefficient & $c_{d}$ \\
wheel inertia & $I_{w}$ & air density & $\rho$ \\
cross-sectional area & $A$ & length & $L$ \\
length of crank & $l_{c}$ & height & $h(x)$ \\
bearing coefficient & $\beta_{0}$ & chain efficiency & $\eta$ \\
bearing coefficient & $\beta_{1}$ & & \\
mechanical gear ratio, bicycle & $\gamma$ & & \\
\hline
\end{tabular}

\section{MODEL-BASED SIMULATION OF CYCLING ON AN ERGOMETER}

Figure 1 shows our bike simulator setup. It consists of a common bicycle frame mounted on an ergometer with an eddy current brake which can be externally controlled by a TCP-IP interface at a rate of $2 \mathrm{~Hz}$. The mathematical model for cycling must be adapted to the ergometer, where the cyclist pedals against the power of the eddy current brake $P_{\text {brake, }}^{\prime}$, the power that causes changes of the kinetic energy of the flywheel $P_{\text {kin }}^{\prime}$, and frictional losses in the chain (factor $\eta$ ), $P_{\text {brake }}^{\prime}+P_{\text {kin }}^{\prime}=\eta P_{\text {ped }}^{\prime}$. Mechanical friction need not be included as it is already accounted for in the internal control of the ergometer brake. Note that primed quantities $(\cdot)^{\prime}$ are used for the ergometer model. Proceeding as before from Equation (1) to (2) we obtain the equation for an equilibrium of forces

$$
F_{\text {brake }}^{\prime}+\frac{I^{\prime}}{r_{w}^{2}} \ddot{x}=\frac{\eta}{\gamma^{\prime}} \frac{l_{c}}{r_{w}} F_{\text {ped }}^{\prime} .
$$

A control software imposes an ergometer brake torque, that simulates the resistance on real courses. For this purpose, the physical models in (2) are used to compute a simulated brake torque that corresponds to the resistance forces barring the inertial force, i.e. $F_{\text {resist }}-F_{\text {kin }}$. The inertial force is partly simulated by the force required to accelerate the ergometer flywheel. However, its moment of inertia $I^{\prime}$ corresponds to a combined inertial mass of cyclist and bicycle of $m_{i}^{\prime}=I^{\prime} / r_{w}^{2}=5,4 \mathrm{~kg}$, which is - as with most ergometers - by far too low. To simulate arbitrary gears without mechanical changes a "virtual gear ratio" is incorporated into the software as follows: To obtain the ergometer brake torque, the simulated brake torque is multiplied with the virtual gear ratio divided by the mechanical gear ratio of the ergometer, which remains fixed at a high value (large gear) at all times. Thus, usually this factor will be larger than 1 . As a consequence, the reciprocal factor must be applied when computing the simulated speed from the mechanical rotation of the

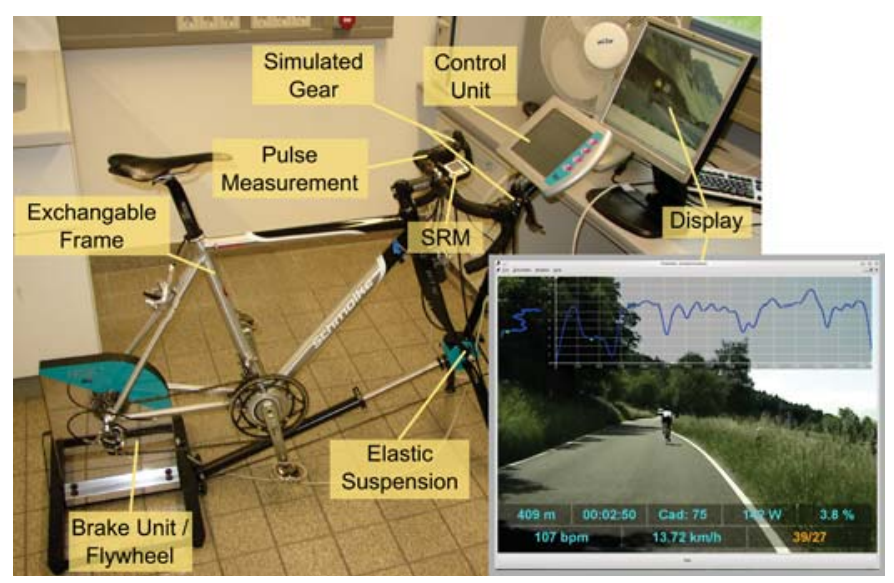

Fig. 1. Our simulator is based on a regular cycle frame and an ergometer with an eddy current brake. The inset image on the lower right shows a frame of the projection display with a registered video frame and current cycling parameters like speed, cadence, power, the road gradient profile and more.

ergometer flywheel. This has two advantages: Firstly, a smaller ratio of required brake torque and angular speed takes load off the eddy current brake and secondly the simulated moment of inertia is closer to realistic values than the physical moment of inertia of the ergometer flywheel.

Two more physical limitations of the ergometer are partly compensated by the simulation software: Firstly, the maximum torque which the eddy current brake can generate is an approximately linear function of the flywheel's angular speed. During the simulation, whenever a higher than possible torque is needed, the simulation software takes over control and decreases the simulated gear, which in turn decreases the ergometer angular speed and reduces the required torque until it is again in the feasible range.

Secondly, the ergometer has no motor, that could accelerate, e.g., when rolling downhill. Moreover, due to the unavoidable mechanical friction, the interface does not allow a nominal brake torque below a certain threshold. When smaller nominal values are required, the simulation software switches to a model-mode, in which the kinetic quantities are computed by the software using the mathematical model and thus are independent of the mechanical ergometer quantities. Thus, to this extent a simulation of accelerating on a downhill section is possible.

To evaluate the simulation precision we compared measurements of power and speed for rides on several uphill courses of about $2-3 \mathrm{~km}$ length and a climb of $200-300 \mathrm{~m}$ with the corresponding simulations on the ergometer in the lab. The riders were instructed to maintain a constant speed or power output. Each pair of corresponding rides was numerically registered and normalized to compensate for small differences of actual speed resp. power during the rides. We found that the differences in power and speed were small, about $1-2 \mathrm{~W}$ mean error in power $(23 \mathrm{~W}$ standard deviation) resp. $0.5-1.0 \mathrm{~km} / \mathrm{h}$ in speed $(1.4-2.4 \mathrm{~km} / \mathrm{h}$ standard deviation). Complete details for the model-based simulation on the ergometer and its evaluation are in Dahmen et al. (2011). It was also argued that the reported 
deviations between field and ergometer measurements can be attributed to the uncertainties in the physical parameters of the model and the slope measurements rather than to the ergometer control.

\section{OPTIMIZATION OF PACING STRATEGIES}

Our second main goal concerns cycling time trials, where a cyclist strives to complete a given course of fixed length $L$ in minimum time $T$. Thus, the performance criterion $\Phi=T$ is to be minimized. A properly posed problem requires to limit the energy resources of the cyclist. The following subsection discusses the problem using fixed mechanical work as the resource constraint, which will be replaced by physiological models in Section 5.2 .

\subsection{Fixed Mechanical Work}

A simple choice for a limited resource is to fix the total amount of mechanical work $E$. The problem is identified as an optimal control problem, with

$$
\dot{x}=v \quad \text { and } \quad \dot{v}=\ddot{x},
$$

where (2) resolved for $\ddot{x}$, yielding the first two model equations. A third state $e(t)$ can be introduced to represent the amount of pedaling work that has been performed:

$$
\dot{e}=P_{\text {ped }} \text {. }
$$

The boundary conditions are

$$
\begin{aligned}
& x(0)=0, \\
& v(0)=v_{0}, \\
& e(0)=0, \\
& x(T)=L, \\
& e(T)=E \text {. }
\end{aligned}
$$

Furthermore, it is necessary to impose restrictions on the pedaling power $P_{\text {ped }}$, which serves as the control variable. Using the box constraints

$$
0<P_{\text {ped }} \leq P_{\text {max }},
$$

with $P_{\max }$ being a fixed maximum power that the cyclist can perform, completes the formulation of the optimal control problem.

To convert such an optimal control problems into a multipoint boundary value problem, it is convenient to introduce the co-states $\lambda_{x}, \lambda_{v}$, and $\lambda_{e}$ and to define the Hamiltonian

$$
\mathcal{H}=\frac{\partial \Phi}{\partial T}+\lambda_{x} \dot{x}+\lambda_{v} \dot{v}+\lambda_{e} \dot{e} .
$$

The three co-state equations $\lambda_{(\cdot)}=-\frac{\partial \mathcal{H}}{\partial(\cdot)}$ and the transversality condition $\lambda_{v}(T)=0$ apply. Pontryagin's Minimum principle gives a necessary condition for the optimal pedaling power $P_{\text {ped }}^{*}$, namely that it minimizes the Hamiltonian:

$$
P_{\text {ped }}^{*}=\arg \min _{P_{\text {ped }}} \mathcal{H} \text {. }
$$

As the control variable $P_{\text {ped }}$ enters linearly into the Hamiltonian, it follows that

$$
P_{\text {ped }}^{*}=\left\{\begin{array}{ll}
P_{\max } & ; S(t)<0 \\
0 & ; S(t)>0
\end{array},\right.
$$

where $S=\frac{\partial \mathcal{H}}{\partial P_{\text {ped }}}$ is the switching function. If $S(t) \equiv 0$ on an interval, then $\dot{S}(t) \equiv 0$ and $\ddot{S}(t) \equiv 0$ provide second order conditions that yield the singular solution

$$
P_{\text {ped }}^{*}=P_{\text {sing }}=F_{\text {resist }} v / \eta ; S=0,
$$

being the third candidate for the optimal pedaling power.
In the special case of cycling on a flat track and starting with a low speed ${ }^{1} v_{0}$, the sequence can be guessed $^{2}$ : $P_{\text {max }} ; P_{\text {sing }} ; 0$. This sequence reflects the intuitive understanding that at the beginning one should accelerate as quickly as possible to reach a certain speed, keep that speed constant for the middle part of the track and decelerate at the end, not to waste kinetic energy when completing the course.

\subsection{Constrained by physiological models}

The idea of fixed work can be replaced by more complex physiological models, as reviewed by Morton (2006), such as the 3-parameter critical power model. This model quantifies the aerobic metabolism, by a maximum (critical) pedaling power $P_{\mathrm{CP}}$ that the cyclist can keep for infinite time. The contribution of the anaerobic metabolism is represented by the state variable

$$
\dot{e}_{\text {an }}=P_{\text {ped }}-P_{\mathrm{CP}},
$$

which replaces (3). The cyclist is exhausted once its maximum, the anaerobic capacity $E_{\text {an }}$, is reached. Instead of (5), the maximum available pedaling power $P_{m}\left(e_{\mathrm{an}}\right)$ is not constant, but decreases linearly with the consumed anaerobic capacity $e_{\text {an }}$ :

$$
0<P_{\text {ped }} \leq P_{m}=P_{\max }\left(1-e_{\mathrm{an}} / E_{\mathrm{an}}\right)
$$

The boundary conditions for $e$ in (4) are replaced by $e_{\text {an }}(0)=0$ and $e_{\text {an }}(T)=E_{\text {an }}$.

Following the procedure in Subsection (5.1), one obtains the optimal power sequence $P_{m} ; P_{\text {sing }} ; P_{m}$ for a long flat track.

Gordon (2005) modifies the model further and computes time to exhaustion $T_{\text {ex }}$ as a function of a constant power output $\bar{P}$ :

$$
T_{\mathrm{ex}}(\bar{P})=\frac{E_{\mathrm{an}}\left(P_{\mathrm{max}}-\bar{P}\right)}{\left(P_{\max }-P_{\mathrm{CP}}\right)\left(\bar{P}-P_{\mathrm{CP}}\right)} .
$$

He generalizes (6) by introducing the exertion state variable $e_{\mathrm{ex}}$ and defines the exertion rate as the reciprocal of time to exhaustion $\dot{e}_{\mathrm{ex}}=T_{\mathrm{ex}}^{-1}(P)$, where variable power $P$ is allowed. Replacing the model equation (3), this definition implies, that for a cyclist that is rested at the beginning and exhausted at the end of the course, the boundary conditions (4) change to $e_{\mathrm{ex}}(0)=0$ and $e_{\mathrm{ex}}(T)=1$. The pedaling power constraint is dropped. The model parameters for a specific cyclist can be estimated by ergometer tests, yet the accuracy of the parameters is limited.

\subsection{Numerical methods and exemplary results}

For the last physiological model, we present numerical results. In Wolf and Dahmen (2010) we used a direct method to solve the optimal control problem. We discretized the states, control variable and the performance criterion and solved the resulting constraint optimization problem with MATLAB's function fmincon(). As an initial guess, we provided $P_{\mathrm{CP}}$ and the corresponding speed by solving the

$1 v_{0}=0$ prohibits a non-vanishing pedaling power.

2 We assume that the track is long enough. 


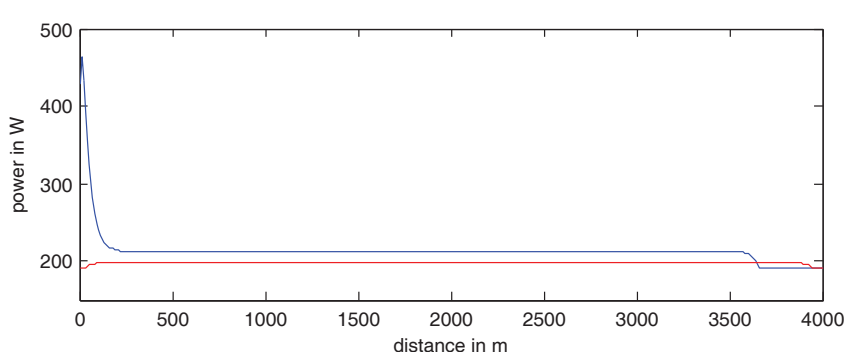

Fig. 2. Optimal pedaling power on a course of $4 \mathrm{~km}$ length with a constant slope of $1 \%$ (blue) and $10 \%$ (red). $P_{\mathrm{CP}}=190 \mathrm{~W}, P_{\max }=1012 \mathrm{~W}$ and $E_{\text {an }}=12225 \mathrm{~J}$

differential equation (2) numerically. This resulted in a significantly reduced computation time for the optimization than if $P_{\mathrm{CP}}$ and a constant velocity were chosen as initial guess.

Figure 2 shows the optimal strategy for a rider on a flat track. Qualitatively, three phases - high power at the beginning, constant power in the middle part and deceleration at the end - can be recognized. The longer the track is, the less pronounced are the high and low power sections at the beginning and the end of the track, respectively.

Wolf and Dahmen (2010) present an example for a simulator ride on a real track (Schienerberg near Radolfzell, Germany), which is similar to the following, depicted in Figure 3. The cyclist first tried to minimize $T$ during a free simulator ride (blue), during which the gradient profile was shown along with the cyclist's current position on the track. As the parameter estimation from simple ergometer tests is not very accurate, the pedaling power and the race time were measured. The final exertion $\tilde{E}_{\text {ex }}$, computed from the measured power, slightly exceeded the theoretical value 1 . Using $\tilde{E}_{\text {ex }}$ as the maximum exertion, we (a) minimized the race time while reaching the same exertion level (red) and (b) minimized the energy expenditure while reaching the same race time (black). In the example of Figure 3 it was possible to reduce the race time in case (a) from 14:09 $\mathrm{min}$ to 13:54 min and the maximum exertion level in case (b) from 1.18 to 0.92 .

With the optimized power from (a) the cyclist could perform a second simulator ride during which he tried to keep the optimal power and speed and finished the track in less time than with the free ride.

\section{PARAMETER CALIBRATION AND HEIGHT PROFILE ESTIMATION}

In this section we describe our model-based estimation of model parameters and of the height profile of the track. Of all model parameters it is the chain efficiency $\eta$, the rolling friction $\mu$, the two bearing friction coefficients $\beta_{0}$ and $\beta_{0}$, the drag coefficient $c_{d}$, and the cross-sectional area $A$ that are difficult to measure directly or to determine in a lab experiment. In addition, they are specific for the cyclist, the bicycle, or the track.

In the model equation (2), the six sought parameters occur in four terms, which differ in their dependency of the pedaling power $P$ and the speed $v$. Therefore, only four
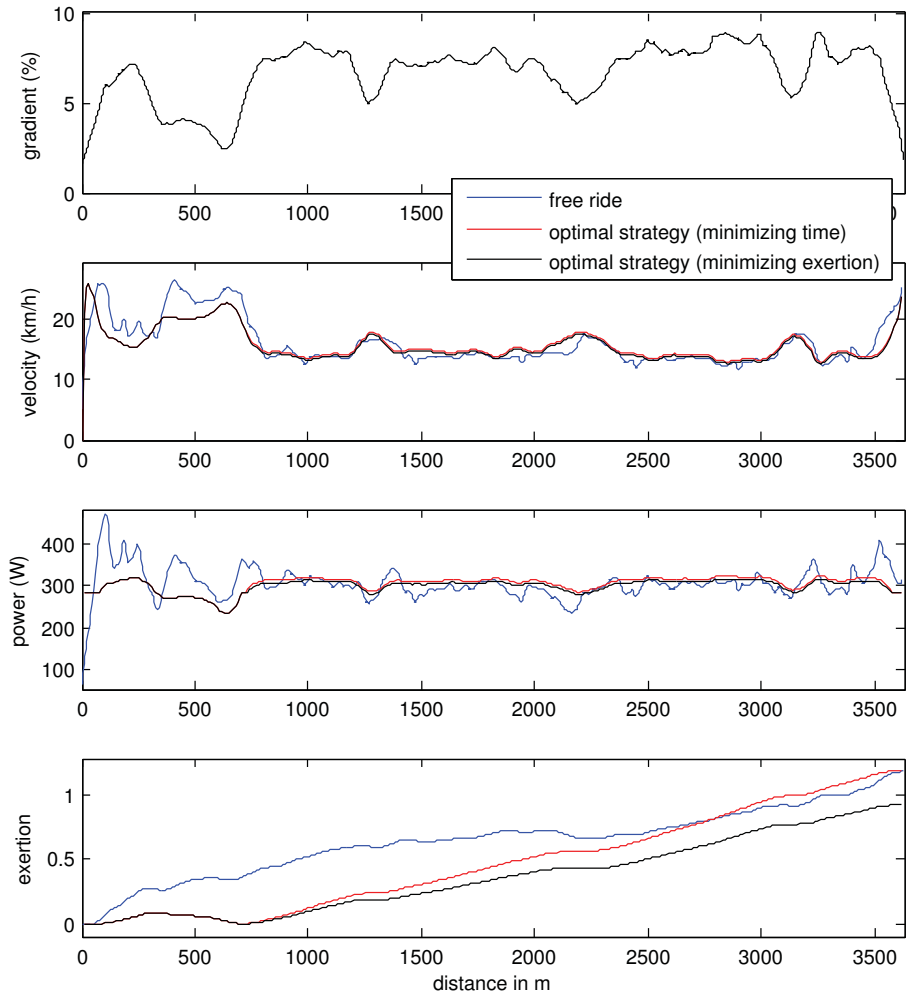

Fig. 3. Optimal strategies vs. free ride. Track: Schienerberg. Rider: $P_{0}=284 \mathrm{~W}, P_{m}=1074 \mathrm{~W}, A=16487 \mathrm{~J}$.

compound coefficients can be estimated based on power and speed measurements. For notational convenience, we stack the four coefficients into the symbolic parameter vector $\boldsymbol{k}$ and the known or measurable constant parameters into a vector $\ell$ :

$$
\boldsymbol{k}=\left(\begin{array}{c}
k_{1} \\
k_{2} \\
k_{3} \\
k_{4}
\end{array}\right)=\left(\begin{array}{c}
1-\eta \\
m g \mu+\beta_{0} \\
\beta_{1} \\
\frac{1}{2} c_{d} \rho A
\end{array}\right) \quad \boldsymbol{\ell}=\left(\begin{array}{c}
\ell_{1} \\
\ell_{2} \\
\ell_{3} \\
\ell_{4}
\end{array}\right)=\left(\begin{array}{c}
g \\
m \\
v_{0} \\
I \\
\frac{r^{2}}{2}
\end{array}\right),
$$

where $v_{0}$ denotes the initial speed. The field measurements are location $x$, speed $v$, and power $P$. We re-parametrize equation (2) with respect to distance in place of time and then rewrite it in terms of $P, v, x$ using $\boldsymbol{k}$ and $\boldsymbol{\ell}$ as follows:

$$
\frac{k_{1} P}{v^{2}}-\left(\ell_{2}+\ell_{4}\right) v^{\prime}(x)-\frac{\ell_{1} \ell_{2} h^{\prime}(x)}{v}-\frac{k_{2}}{v}-k_{3}-k_{4} v=0,
$$

subject to $\left.v(x)\right|_{x=0}-\ell_{3}=0$. We interpret (7) as an implicit definition of the speed function

$$
v:[0, L] ; \mathbb{R}_{+}, \quad x \mapsto v\left(x ; \boldsymbol{k}, \boldsymbol{\ell}, h^{\prime}(\cdot), P(\cdot)\right),
$$

where $L$ is the length of the track.

Given a set of known or measured parameters $\boldsymbol{\ell}$, functions $h^{\prime}(\cdot), P(\cdot)$, and speed measurements $v_{m}(x)$ for a specific ride of a cyclist on the given track, our approach is to seek parameters $\boldsymbol{k}$, so that $v(x ; \boldsymbol{k})$ is close to the speed measurement $v_{m}(x)$. Therefore, we define a cost function

$$
J(\boldsymbol{k})=\frac{1}{L} \int_{0}^{L}\left(v\left(x ; \boldsymbol{k}, \boldsymbol{\ell}, h^{\prime}(\cdot), P(\cdot)\right)-v_{m}(x)\right)^{2} \mathrm{~d} x
$$

as the mean squared deviation of the modeled speed from the measurement over the length of the track. Then, we determine the estimated parameter vector as $\hat{\boldsymbol{k}}=$ $\arg \min _{\boldsymbol{k}} J(\boldsymbol{k})$. Such optimization problems can be solved numerically by gradient-based methods. 
In contrast to previous model-based methods our approach allows to make measurements on tracks that need not be perfectly flat and where the speed, resp. power output of the rider need not be constant. This removed some of the uncertainty in previous model-based parameter estimation methods. For numerical results, an empirical validation, an analysis of the sensitivities of the parameters, and limitations of the method see Dahmen and Saupe (2011).

For the above parameter estimation precise altitude resp. slope data for the used track for the measurements was assumed available if the selected track was not ideally flat. Such data should be obtained from precise differential GPS measurements. In the simulator and for performance optimization the model will yield high accuracy predictions for uneven tracks only if precise elevation and gradient data is also provided for the entire track. As mentioned, differential GPS may fail in road intervals with obstacles like trees and houses along the side. To overcome this problem, we propose to use model-based slope estimates by resolving the model equation (2) for $\frac{\mathrm{d} h}{\mathrm{~d} x}$ and evaluating it using the measured power $P(x)$ and speed $v(x)$ at distances $x$. In order to avoid drifting artifacts, the distances $x$ should not be taken from distances given by a bike computer. Instead, all measurement locations are obtained from projecting its longitude and latitude GPS coordinates on a previously generated cubic reference spline of the road. Such a cubic spline can be obtained from a separate GPS measurement or from geographical survey data. As the method estimates only the slope rather than the altitude, the slope is integrated, and the resulting altitude function was scaled and shifted, so that the resulting altitude is continuous at the boundaries of the intervals where precise differential GPS data is available, again. Finally, $h(x)$ is computed as a smoothed cubic spline minimizing a functional that adds sums of weighted height differences and the mean squared second derivatives of the height function.

A comparison of this method to the slope estimate $\frac{\mathrm{d} h_{\mathrm{dgps}}}{\mathrm{d} x}$ on a section of the track, where precise differential GPS data were available, showed that this slope estimation is more accurate than the slope estimate $\frac{\mathrm{d} h_{\mathrm{gps}}}{\mathrm{d} x}$ obtained by differentiating the standard GPS altitude data. For more details see Dahmen et al. (2011).

\section{CONCLUSION}

Mathematical modeling brings about a number of benefits for sport science that would otherwise be hard to obtain if not impossible. In three use cases we showed that state-ofthe-art mechanical modeling of cycling and a simple physiological model of human endurance performance supplies the foundation of an accurate cycling simulator in the laboratory, and provides optimal pacing strategies for athletes on uphill time trials. Moreover, the models allow to infer precise values of their parameters from experiments in the field rather than from elaborate direct measurements or complicated simulations. This includes exact height profiles of cycling tracks that are not available at the required precision from geographical surveys and that cannot be measured everywhere by high precision differential GPS.

\section{REFERENCES}

Andreeva, E. and Behncke, H. (1998). Competitive running on a hilly track. International Series of Numerical Mathematics, 241-250.

Atkinson, G. and Brunskill, A. (2000). Pacing strategies during a cycling time trial with simulated headwinds and tailwinds. Ergonomics, 43(10), 1449-1460.

Atkinson, G., Peacock, O., and Passfield, L. (2007). Variable versus constant power strategies during cycling time-trials: Prediction of time savings using an up-todate mathematical model. Journal of Sports Sciences, 25(9), 1001-1009.

Behncke, H. (1997). Optimization models for the force and energy in competitive running. Journal of Mathematical Biology, 35(4), 375-390.

Dahmen, T., Byshko, R., Saupe, D., Röder, M., and Mantler, S. (2011). Validation of a model and a simulator for road cycling on real tracks. Sports Engineering, 14, 95-110.

Dahmen, T. and Saupe, D. (2011). Calibration of a powerspeed-model for road cycling using real power and height data. International Journal of Computer Science in Sport, 10(2), 18-36.

Defraeye, T., Blocken, B., Koninckx, E., Hespel, P., and Carmeliet, J. (2010). Aerodynamic study of different cyclist positions: CFD analysis and full-scale windtunnel tests. Journal of Biomechanics, 43(7), 12621268 .

di Prampero, P.E., Cortili, G., Mognoni, P., and Saibene, F. (1979). Equation of motion of a cyclist. Journal of Applied Physiology, 47(1), 201-206.

Gordon, S. (2005). Optimising distribution of power during a cycling time trial. Sports Engineering, 8(2), 81-90.

Lim, A., Homestead, E., Edwards, A., Carver, T., Kram, R., and Byrnes, W. (2011). Measuring changes in aerodynamic/rolling resistances by cycle-mounted power meters. Medicine and Science in Sports and Exercise, 43(5), 853-860.

Maronski, R. (1994). On optimal velocity during cycling. Journal of Biomechanics, 27(2), 205-213.

Martin, J., Gardner, A., Barras, M., and Martin, D. (2006). Modeling sprint cycling using field-derived parameters and forward integration. Medicine and Science in Sports and Exercise, 38(3), 592-597.

Martin, J., Milliken, D., Cobb, J., McFadden, K., and Coggan, A. (1998). Validation of a mathematical model for road cycling power. Journal of Applied Biomechanics, 14, 276-291.

Morton, R. (2006). The critical power and related wholebody bioenergetic models. European Journal of Applied Physiology, 96(4), 339-354.

Olds, T.S., Norton, K.I., Lowe, E.L., Olive, S., Reay, F., and Ly, S. (1995). Modeling road-cycling performance. Journal of Applied Physiology, 78(4), 1596-1611.

Olds, T., Norton, K., and Craig, N. (1993). Mathematical model of cycling performance. Journal of Applied Physiology, 75(2), 730-737.

Wolf, S. and Dahmen, T. (2010). Optimierung der Geschwindigkeitssteuerung bei Zeitfahrten im Radsport. In D. Link and J. Wiemeyer (eds.), Sportinformatik trifft Sporttechnologie, 235-239. dvs-Sektion Sportinformatik. 